\title{
Radioimmunoassay zum Nachweis von Hepatitis B (HB, Australia)- Antigen im Serum
}

\author{
Von U. Zeidler, B. Baruth, H. Deicher, E. Hultsch, M. Kuse, R. Müller und W. Wittwer
}

\author{
Aus dem Institut für Nuklearmedizin und spezielle Biopbysik (Prof. Dr. H. Hundesbagen) Department Radiologie, der \\ Abteilung Biometrie (Prof. Dr. B. Scbneider) Department für Medizinische Informatik und Biometrie, der Abteilung für \\ Gastroenterologie und der Abteilung für Klinische Immunologie des Departments Innere Medizin \\ der Medizinischen Hocbscbule Hannover
}

(Eingegangen am 2. Juni/7. September 1973)

An Seren von 321 Patienten wurde nach Vielfachbestimmungen durch hierarchische Varianzanalyse ein solid-phase Radioimmunoassay (125I-Ausria, Abbott Lab. Inc.) hinsichtlich Präzision, Fehlermöglichkeiten und Verbesserungsmöglichkeiten geprüft. Zur besseren Vergleichbarkeit der Ergebnisse, die mit unterschiedlichen technischen Ausrüstungen gewonnen werden, erfolgt die Angabe der Entscheidung: „Serum antigenhaltig“ oder „Antigen nicht nachweisbar“ in Form eines Quotienten aus der Zählrate des Probandenserums und dem „Negativwert“ der jeweiligen Serie (RIA-Faktor). Anstelle eines willkürlichen Grenzwertes für die Entscheidung „Serum antigenhaltig" wurde mit Hilfe von Seren von Patienten, die an einer Hepatitis B mit transienter Antigenämie erkrankt waren und zum Zeitpunkt der letzten Antigenbestimmung weder nach klinischen, laborchemischen und histologischen Gesichtspunkten Zeichen eines Leberschadens aufwiesen, ein „Normbereich“ ermittelt (Mittelwert 1,2;s $=0,2$ ).

Der durch Vielfachbestimmungen an verschiedenen Patientenseren ermittelte Gesamtfehler der Methode beträgt bei Doppelbestimmungen $\pm 16 \%$. Der relativ hohe Variationskoeffizient schränkt jedoch die Differenzierungsqualität des Verfahrens nicht entscheidend ein.

Verlaufsbeobachtungen an Patientenseren mit Antigenämie und Verdünnungsreihen ließen erkennen, daß nur in einem eng begrenzten Bereich ein lineares Verhältnis zwischen Antigenkonzentration im Serum und Höhe des RIA-Faktors besteht. Die relative Empfindlichkeit des Testansatzes im Vergleich zur Komplementbindungsteaktion lag mit einer im Mittel 32-64-fach höheren Empfindlichkeit in der Größenordnung anderer radioimmunologischer Verfahren. Die Menge des eingesetzten Probandenserums und die des zugefügten radioaktiven Antikörpers waren im Sättigungsbereich der Methode und bei antigenfreien Seren ohne Einfluß auf das Testergebnis. Die in dieser Untersuchung eingesetzten Pharmaka zeigten keinen Einfluß auf die Meßergebnisse. Bei Patienten mit Rheumafaktor im Serum, die an einer Hepatitis B erkrankten, war die Antigenämie in jedem untersuchten Fall nachweisbar.

Eindeutig zeigte sich eine Abhängigkeit der Testergebnisse von der Inkubationsdauer und der Inkubationstemperatur. Untersuchungen an 30 Seren unterschiedlicher Antigenkonzentration lassen erkennen, daß die Zeit der Erstinkubation ohne Empfindlichkeitsverlust auf $3 \mathrm{~h}$ verkürzt werden kann, wenn gleichzeitig die Inkubationstemperatur (hier $37^{\circ} \mathrm{C}$ ) erhöht wird.

\section{Radioimmuno-assay for the detection of $H B$ (Australia)-antigen in sera}

The precision, reliability and possible improvements of a solid-phase radioimmuno-assay ( ${ }^{225}$ I-Ausria, Abbott Lab. Inc.) have been tested by a statistical analysis of variance, using sera from 321 patients with and without antigen.

For a comparison of results obtained with different equipment in different laboratories the results are obtained as the ratio of the count rate of the patient's serum and the negative control value. Instead of postulating an arbitrary "cut-off value" a "normal range" has been estimated on "antigen-free" sera of patients that suffered from Hepatitis B with transient antigenemia and were judged as recovered completely on the basis of clinical, enzymatic and histological criteria. The mean value obtained was 1.2 with a variation coefficient of $16 \%$. The results confirm, that to use a "cut-off value" of twice the negative value is a practicable procedure although this hypothesis has to be backed by quantitative determinations in future. Carrying out double and triple examinations the overall error of the method was $\pm 16 \%$ for double examinations. The value was obtained using different patient's sera instead of using a single standard serum. Follow-up studies and dilution of antigen-containing sera revealed a small range of linearity only. The relative sensitivity of the method as compared to the complement fixation test was comparable to other radioimmuno techniques. Neither the amount of patient's serum added nor the amount of radioactive antibody added showed any influence on the results as far as negative results and values in the saturation range are concerned. The pharmaceuticals examined as well as a positive Rheumafaktor did not change the test results.

There was a definite dependancy of the test results on preincubation time and incubation temperature. It can be stated that the first incubation stage may be reduced to as little as $3 \mathrm{~h}$, provided the incubation temperature is raised $\left(37^{\circ} \mathrm{C}\right.$ in these experiments) during this time.

Die Bedeutung des HB-Antigens als spezifisches Indikator-Antigen für eine Infektion mit dem Erreger der Virushepatitis $B$ wird heute allgemein anerkannt und ist durch zahlreiche Untersuchungen eindeutig belegt (1-5). Der Nachweis des Antigens im Verlauf einer Erkrankung mit Gelbsucht ist für die Differentialdiagnose, Prognose und Prophylaxe der Virushepatitis sowie für epidemiologische Studien von hohem Wert $(6-20)$.
Nachweisverfahren für HB-Antigen müssen daher neben Spezifität höchstmögliche Empfindlichkeit aufweisen und durch geringen technischen Aufwand oder die Möglichkeit zur Automatisation rasche und zuverlässige Bestimmungen auch in größeren Untersuchungsreihen bieten.

Die ursprünglich für den Nachweis des HB-Antigens eingesetzten Methoden entsprechen den genannten 
Forderungen in dem einen oder dem anderen Punkt nur unvollkommen $(21-29)$.

Derzeit höchstmögliche Empfindlichkeit erreichen die radioimmunologischen Techniken (30-37), von denen das nach dem solid-phase Prinzip (38) arbeitende Verfahren durch einfache Handhabung und durch die Möglichkeit zumindest teilweiser Automatisierung die Untersuchung auch größerer Probenzahlen erlaubt.

Radioimmunologische Methoden haben zudem den Vorteil der digitalen Auswertung der Meßergebnisse. Sie sind damit frei von eventuellen Fehlern subjektiver Beurteilung und statistischen Analysen im Hinblick auf Präzision der Untersuchung und Qualitätskontrollen zugänglich (39). Die in diesem Bericht vorgelegten Ergebnisse geben einen Überblick über die Präzision, die Empfindlichkeit, den erforderlichen Zeitaufwand und die Fehlermöglichkeiten eines inzwischen kommerziell erhältlichen (125I-Ausria, Abbott Lab. Inc.), nach dem solid-phase Prinzip aufgebauten Testsatzes zum Nachweis von Hepatitis B-Antigen im Serum.

\section{Material und Methoden}

Der Testsatz ${ }^{125}$ I-A Ausria besteht aus Polypropylen-Röhrchen, die entsprechend dem solid-phase Verfahren mit einem von Meerschweinchen gewonnenen, gegen menschliches HB-Antigen gerichteten Antikörper unbekannter Subtypen beschichtet sind. Die Röhrchen werden mit $0,1 \mathrm{ml}$ des zu untersuchenden Serums beschickt und für $16 \mathrm{~h}$ bei Raumtemperatur inkubiert. Dabei wird eventuell im Serum vorhandenes Antigen durch den Antikörper gebunden. Durch mehrmalige Waschungen werden Serum und nicht gebundenes Antigen entfernt und anschließend radioaktiv markierte Antikörper unbekannter spezifischer Aktivität vom Meerschweinchen in standardisierter Menge $(0,1 \mathrm{ml})$ zugefügt. Nach 90 min Inkubation bei Raumtemperatur wird der nicht von dem eventuell vorhandenen, polyvalenten Antigen gebundene radioaktive Antikörper durch erneute Waschvorgänge entfernt und die Radioaktivität im Teströhrchen gemessen. Die Höhe der meßbaren Impulstate ist innerhalb eines bislang nicht sicher definierten Bereiches ein relatives $\mathrm{Ma}_{a} \beta$ für die Menge fixierten Antigens.

$\mathrm{Da}$ es auch obne Vorhandensein von HB-Antigen im Probandenserum, wahrscheinlich durch unspezifische Anlagerung des radioaktiven Antikörpers an die Wand des Testgefäßes oder an den im Röhrchen befindlichen Antikörper zu einer über den Nullwert des Teströhtchens hinausgehenden meßbaren Impulstate kommt, muß in jeder Serie ein sogenannter "Negativwert" dadurch exmittelt werden, daß Teströhrchen mit einem vom Hersteller als sicher HB-antigenfrei deklarierten Kontrollserums beschickt, mit dem radioaktiv markierten Antikörper inkubiert und den gleichen Wasch- und Meßvorgängen unterzogen werden. In diesen Untersuchungen wurde jeweils der Mittelwert aus fünf Bestimmungen als „Negativivert" für die Errechnung des RIA-Faktors benutzt. Zur Kontrolle der Funktionsfähigkeit des Testsatzes wird vom Hersteller ein antigenhaltiges Serum unterschiedlicher Konzentration geliefert, das bei korrekter Technik eine Impulsrate von mehr als dem Doppelten des „Negativwertes“ ergeben muß.

Die Angabe der Impulsrate (Imp./min) sagt ohne gleichzeitige Angabe des „Negativwertes" der entsprechenden Serie nichts über die Höhe der Antigenkonzentration im Serum aus. $\mathrm{D}_{2}$ die gemessene Impulsrate zudem von den Gerätceigenschaften wie Kristalldurchmesser des Szintillationszählets, Einstellung der Diskriminatoren usw. abhängig ist, empfiehlt es sich, für den Nachweis von Trends bei Verlaufsbeobachtungen und für den Vergleich der Ergebnisse verschiedener Laboratorien einen unabhängigen Faktor anzugeben.
Hier wurde dafür der Quotient aus der Impulsrate des Probandenserums und dem „Negativwert" der jeweiligen Serie gewählt und dieser als Radio-Immuno-Assay Faktor (RIA-Faktor) bezeichnet:

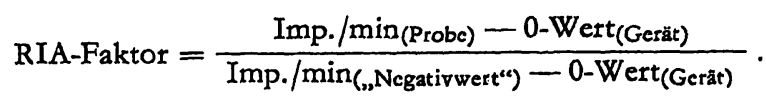

Dieser Faktor müßte bei antigenfreiem Serum $=1,0$ sein. Bedingt durch die zufälligen Fiehler der Methode, besteht eine größere Streubreite, so daß ein „Normbereich" angenommen werden muß.

Die dem Verfahren anhaftenden Fehlermöglichkeiten reichen von der Möglichkeit wechselnden Antikörpergehaltes der Teströhrchen über unzureichende Waschvorgänge bis zu Instabilitäten des Meßsystems. Die Febler der einzelnen Arbeitsschritte addieren sich nach dem Fehlerfortpflanzungsgesetz zum Gesamtfehlex der Methode, der ein Maß für die Präzision des Verfahrens darstellt. Während über den systematischen Fehler der Methode durch Nachweis der Empfindlichkeitsschwelle hier keine Aussage gemacht werden kann, da zum Zeitpunkt der Untersuchungen kein Serum mit definierter Menge Antigenpartikel zur Verfügung stand, wurde die Reproduzierbarkeit für die folgenden methodischen Verfahrensweisen ermittelt: Für den „Negativwert“, für Bestimmungen in der Serie, für Bestimmungen von Tag zu Tag mit gleichen Chargen und gleichen Chemikalien und für Bestimmungen von Tag zu Tag mit unterschiedlichen Chargen und Chemikalien.

\section{Statistische Methoden}

Um die Untersuchungen den Gegebenheiten des Routinebetriebes anzupassen, erfolgte die Prüfung nicht an Standardseren wie für die Qualitätskontrolle, sondern anhand der aus Doppel- und Dreifachbestimmungen von Probandenseren gewonnenen Meßergebnisse.

Der Bestimmung der Präzision der „Negativwerte" und den Bestimmungen in der Serie wurden die gemesserien Impulsraten (Imp./10 min) zugrunde gelegt.

Im einstufigen hierarchischen Versuchsplan wurde der Variationskoeffizient (VK) für die Einzelbestimmung über die Varianzen der Mehrfachbestimmungen an einem Serum und von Serum zu Serum ermittelt: Es wurden $N$ Seren untersucht. Am i-ten Serum wurden $n_{1}$-Bestimmungen zum Nachweis einer Antigenämie durchgeführt. Dann ist $\mathrm{y}_{\mathrm{ij}}$ das Ergebnis der $\mathrm{j}$-ten Bestimmung am Serum des i-ten Patienten.

In dem Modell: $y_{i j}=\mu+a_{i}+\varepsilon_{i j}$, ist $\mu$ der wahre Meßwert, $a_{i}$ $\operatorname{der}$ Einfluß des Einzelpatienten der hier zu vernachlässigen ist, und $\varepsilon_{\mathrm{ij}}$ der Fehler in der j-ten Bestimmung bei dem i-ten Patienten. $\varepsilon_{i j}$ sei zufällig normalverteilt mit dem Mittelwert 0 . Die Varianz der Grundgesamtheit des Feblers $\varepsilon_{\mathrm{ij}}\left(\sigma^{2}\right)$ läßt sich dann durch die Varianz det Stichprobe $\left(\mathrm{s}^{2}\right)$ schätzen:

$$
\begin{gathered}
\mathrm{n}=\sum_{\mathrm{i}=1}^{N} \mathrm{n}_{\mathrm{i}} \\
\overline{\mathrm{y}}_{\mathrm{i}}=\frac{1}{\mathrm{n}_{1}} \sum_{j=1}^{\mathrm{n}_{1}} \mathrm{y}_{i j} \\
\mathrm{~s}^{2}=\frac{1}{\mathrm{n}-\mathrm{N}} \sum_{\mathrm{i}=1}^{N} \sum_{j=1}^{\mathrm{n}_{\mathrm{i}}}\left(\mathrm{y}_{i j}-\overline{\mathrm{y}}_{\mathrm{i}}\right)^{2}
\end{gathered}
$$

Führt man nur eine Doppelbestimmung durch, dann vereinfacht sich die Formel auf:

$$
s^{2}=\frac{1}{2 N} \sum_{i=1}^{N}\left(y_{i 1}-y_{i_{2}}\right)^{2}
$$

Zu dem erbaltenen Fehler der Messung des Probandenserums $\left(x_{1}\right)$ ist der Fehler aus der Bestimmung der "Negativwerte“ $\left(x_{2}\right)$ zu addieren, da der RIA-Faktor ( $y$ ) aus beiden Werten gebildet wird:

$$
\mathrm{y}=\frac{\mathrm{x}_{1}}{\mathrm{x}_{2}}
$$


Für den relativen Fehler $\Delta y$ des RIA-Faktors ergibt sich nach dem Fehlerfortpflanzungsgesetz:

$$
|\Delta \mathrm{y}|=\left|\frac{1}{\mathrm{X}} \Delta \mathrm{x}_{1}\right|+\left|\frac{\mathrm{X}_{1}}{\mathrm{X}_{2}} \Delta \mathrm{X}_{2}\right|
$$

und nach einigen Umformungen:

$$
\left|\frac{\Delta \mathrm{y}}{\mathrm{y}}\right|=\left|\frac{\Delta \mathrm{X}_{1}}{\mathrm{X}_{1}}\right|+\left|\frac{\Delta \mathrm{x}_{2}}{\mathrm{X}_{2}}\right|
$$

Zur Interpretation der Ergebnisse nach Erweiterung der Fehlerquellen, Präzision von Tag zu Tag und Präzision bei wechselnden Chargen und Chemikalien, ist zu beachten, daß für den jeweils hinzu kommenden Fehler nicht die Differenz zwischen der Standardabivcichung $s$ des ersten Versuches und der Standardabweichung $s_{1}$ des erweiterten Versuches verwendet wurden, sondern daß

$$
s_{2}=\sqrt{s_{1}^{2}-s^{2}}
$$

ist. Gleiches gilt für den Variationskocffizienten.

$\mathrm{Da}$ der Variationskoeffizient in den Untersuchungen zur Präzision in der Serie in allen Untergruppen annähernd gleich war, wurde wegen der geringen Fallzahlen eine Gruppenunterteilung für die Bestimmung der Präzision von Tag zu Tag nicht vorgenommen. In diesen Untersuchungen wurde außerdem anstelle der Impulsraten der RIA-Faktor als Grundlage der Berechnungen verwendet.

Für die Untersuchungen der Zeit- und Temperaturabhängigkeit des Verfahrens wurden zweistufige hierarchische Versuchspläne angelegt. Der RIA-Faktor jedes Patientenserums wurde in jeder modifizierten Form, jedoch immer gleich oft bestimmt. Für jede Untersuchungsform wurde die „Reproduzicrbarkeitsstreuung“ getrennt berechnet und unter Voraussetzung, daß diese sich nicht unterscheiden, eine gemeinsame Schätzung vorgenommen. Unter der 0-Hypothese, daß zwei zu vergleichende Untcrsuchungsgänge sich nicht unterscheiden, unterschiedliche Meßwerte also nur durch „Reproduzierbarkeitsstreuung" bedingt sind, wurde dann eine weitere, von der ersten Schätzung unabhängige Schätzung der „Reproduzierbarkeitsstreuung“ vorgenommen und durch die Beziehung:

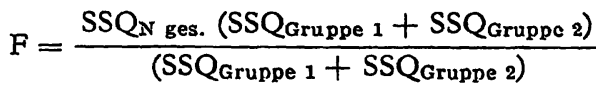

$$
\frac{\left(\mathrm{N}_{\text {Gruppe 1 }}+\mathrm{N}_{\text {Gruppe 2 }}\right)}{\mathrm{N}_{\text {ges. }}-\left(\mathrm{N}_{\text {Gruppe } 1}+\mathrm{N}_{\text {Gruppe 2 }}\right)}
$$

anhand des F-Test geprüft, ob die Hypothese, daß beide Varianzen gleich sind, zu verwerfen ist $(40,41)$.

\section{Ergebnisse}

Präzision der „Negativwerte“ von Tag zu Tag in verschiedenen Ansätzen

Der „Negativwert" bildet die Basis für die Entscheidung, ob ein Serum als antigenhaltig oder nicht antigenhaltig bezeichnet wird, gleichgültig, ob für die Entscheidung die Überscbreitung eines willkürlich gesetzten Grenzwertes oder ob die Erhöhung des Quotienten aus der Impulsrate des Probandenserums und der Impulstate des „Negativwertes“ zugrunde gelegt urird.

Die „Negativwerte" wurden in 41 Bestimmungen zu jeweils 5 Werten ermittelt und die Schwankung der Meßwerte um den unbekannten wahren Wert mit Hilfe der einstufigen Varianzanalyse geschätzt.

Dabei ergaben sich für die Bestimmungen von Tag zu Tag unter Wechsel der Chemikalien und Chargen die in Tabelle 1 aufgeführten Werte für die Präzision einer Einzelmessung. Da der „Negativwert" für eine Serie jedoch aus jeweils fünf Bestimmungen ermittelt wurde,
Tab. 1

Präzision des „Negativwertes", ermittelt an 41 Kontrollseren bei jeweils 5 Bestimmungen (Angabe in $1 \mathrm{mp} . / 10 \mathrm{~min}$ )

\begin{tabular}{cccc}
\hline $\mathrm{n}$ & $\overline{\mathrm{x}}$ & $\mathbf{s}^{2}$ & $\mathrm{VK}$ \\
\hline 205 & 4478 & 225718 & $10,0 \%$ \\
\hline
\end{tabular}

beträgt der zu den weiteren Bestimmungen zu addierende Fehler nur $\frac{10}{1 / 5}=4,5 \%$. Dieser Fehler gilt für eine Meßzeit von $10 \mathrm{~min}$, die aus Gründen der raschen Durchführbarkeit gewählt wurde. Bei Verlängerung der Meßzeiten läßt sich dieser Fehler weiter reduzieren.

\section{Präzision in der Serie}

Für diese Untersuchungen wurden die Meßwerte nach der Höhe der Zählraten in drei Gruppen geordnet, um mögliche Beeinflussungen durch Einzelkomponenten erkennbar werden zu lassen:

\section{Gruppe 1}

$\mathrm{n}=152$

Ansätze, bei denen die gemessenen Impulstaten zwischen 4-8000 Imp./10 min lagen, entsprechend einem RIAFaktor kleiner als 2,0.

\section{Gruppe 2}

$\mathrm{n}=82$

Ansätze, die Impulsraten zwischen 8000 und $75000 \mathrm{Imp}$./ $10 \mathrm{~min}$ ergaben, entsprechend einem RIA-Faktor zwischen 2,0 und 15,0 .

\section{Gruppe 3}

$\mathrm{n}=87$

Ansätze mit Impulsraten von mehr als $75000 \mathrm{Imp}$./ $10 \mathrm{~min}$ entsprechend einem RIA-Faktor von über 15,0.

In allen Gruppen wurde durch die gleichzeitige Bestimmung der $\mathrm{F}$-Werte nachgewiesen, daß die Methode nicht nur zufällig differenziert.

Die durch statistische Analyse ermittelten Werte für die Variationskoeffizienten der einzelnen Gruppen sind in Tabelle 2 aufgeführt.

Die Variationskoeffizienten der einzelnen Gruppen unterscheiden sich nicht wesentlich voneinander, obwohl die Mittelwerte der Impulsraten zwischen den Gruppen 1 und 3 um etwa den Faktor 10 differieren. Dies deutet darauf hin, daß die durch höhere Zählraten zu erwartende verbesserte Präzision durch andere, neu

Tab. 2

Präzision in der Serie bei Untersuchungen zum Nachweis des HBAntigens, ermittelt an antigenfreien und antigenhaltigen Patientenseren. (Angaben in $I \mathrm{mp} . / 10 \mathrm{~min}$ )

\begin{tabular}{lrrrr}
\hline & $\mathrm{n}$ & \multicolumn{1}{c}{$\overline{\mathrm{x}}$} & \multicolumn{1}{c}{$\mathrm{s}^{3}$} & \multicolumn{1}{c}{$\mathrm{VK}$} \\
\hline Gruppe 1 & 152 & 4784 & 231692 & $10,0 \%$ \\
Gruppe 2 & 82 & 36064 & 12380476 & $9,7 \%$ \\
Gruppe 3 & 83 & 92830 & 54389223 & $7,9 \%$ \\
\hline
\end{tabular}


Tab. 3

Verbesserung der Präzision in der Serie bei Bestimmungen zum Nachweis von HB-Antigen im Serum durch Mehrfachuntersuchungen

\begin{tabular}{cccc}
\hline & \multicolumn{2}{c}{ Variationskoeffizient [\%] bei } \\
& Einfach- & $\begin{array}{c}\text { Vierfach- } \\
\text { Doppel- } \\
\text { bestimmung }\end{array}$ \\
\hline Gruppe 1 & 10,0 & 7,1 & 5,0 \\
Gruppe 2 & 9,7 & 6,9 & 4,9 \\
Gruppe 3 & 7,9 & 5,6 & 4,0 \\
\hline
\end{tabular}

Tab. 4

Gesamtfehler der Methode zur Bestimmung von HB-Antigen im Serum bei Anwendung in der Serie und unter Berücksichtigung des Präzisionsfehlers des "Negativwertes" sowie der Möglichkeit von Mehrfachbestimmungen

\begin{tabular}{lccc}
\hline & \multicolumn{2}{c}{ Variationskoeffizient [\%] bei } \\
Einfach- & Doppel- & $\begin{array}{c}\text { Vierfach- } \\
\text { bestimmung }\end{array}$ \\
\hline Gruppe 1 & 14,5 & 11,6 & 9,5 \\
Gruppe 2 & 14,2 & 11,4 & 9,3 \\
Gruppe 3 & 12,3 & 10,1 & 8,5 \\
\hline
\end{tabular}

hinzu tretende Fehler beeinträchtigt wird. Insgesamt liegen die ermittelten Variationskoeffizienten bedeutend höher als, sie beispielsweise bei routinemäßigen Autoanalyzerqualitätskontrollen zur Bestimmung von Kationen im Serum gefunden werden und übersteigen auch Werte, wie sie für Enzymbestimmungen im Serum angegeben werden. Es ist hier jedoch zu berücksichtigen, $\mathrm{da} \beta$ die ermittelten Werte nicht aus Bestimmungen an einem Standardserum stammen, sondern daß Grundlage der Berechnungen wiederholte Bestimmungen bei insgesamt 321 Patientenseren ist. Der angegebene Fehler gilt für eine Einfachbestimmung und eine Wahrscheinlichkeit von $67 \%$.

Der Fehler wird entsprechend kleiner, wenn für die Bestimmung des RIA-Faktors der Mittelwert aus mehreren Ansätzen herangezogen wird. Er reduziert sich dann auf den durch die Wurzel der Anzahl der Bestimmungen dividierten Variationskoeffizienten der Einzelbestimmung (Tab. 3).

Den Gesamtfehler erhält man durch Addition des Fehlers, der bei Bestimmung der „Negativwerte“ entsteht und unter Zugrundelegung der 5-fach Bestimmung hier $4,5 \%$ beträgt, so daß die in Tabelle 4 wiedergegebenen Werte resultieren.

Präzision von Tag zu Tag bei gleicher Charge und gleichen Chemikalien

Die Präzision von Tag zu Tag wurde an 35 antigenhaltigen Seren ermittelt, indem an verschiedenen Tagen die RIA-Faktoren aus dem Mittelwert einer Doppelbestimmung unter Zugrundelegung jeweils neu be-

Tab. 5

Präzision der Methode zur Bestimmung von HB-Antigen im Serum bei Bestimmungen von Tag zu Tag und Verwendung gleicher Testsätze (Angaben als RIA-Faktor)

\begin{tabular}{lllll}
\hline $\mathrm{n}$ & $\overline{\mathbf{x}}$ & $\mathrm{s}^{2}$ & VK \\
\hline 35 & 16,2 & 4,5 & $13 \%$ \\
\hline
\end{tabular}

stimmter "Negativwerte“ errechnet und untereinander verglichen wurden. Gegenüber den Ergebnissen in der Serie verringert sich hier die Präzision von 11,6 auf $13 \%$ für Doppelbestimmungen (Tab. 5).

Präzision von Tag zu Tag unter Verwendung neuer Chargen und nẹuer Chemikalien

In der routinemäßigen Durchführung der Untersuchungen wird die Präzision durch zusätzliche Faktoren wie Qualitätsunterschiede oder Änderungen der verwendeten Chargen, Chemikalien oder Untersuchungsbedingungen beeinflußt. Das Ausmaß dieser Einflüsse wurde durch wiederholte Doppelbestimmungen unter Verwendung jeweils neuer Chargen und Chemikalien an 45 antigenhaltigen Seren untersucht. Erwartungsgemäß muß eine weitere Minderung der Präzision in Kauf genommen werden (Tab. 6).

Tab. 6

Präzision der Methode zur Bestimmung von HB-Antigen im Serum bei Bestimmungen von Tag zu Tag und wechselnden Testsätzen (Angaben als RIA-Faktor)

\begin{tabular}{rllll}
\hline $\mathrm{n}$ & $\overline{\mathrm{x}}$ & $\mathrm{s}^{2}$ & $\mathrm{VK}$ \\
\hline 45 & 17,9 & 8,2 & $16 \%$ \\
\hline
\end{tabular}

Der „Normbereich“

Über die absolute Nachweisempfindlichkeit der verschiedenen Methoden zur Bestimmung von $\mathrm{HB}$ Antigen im Serum ist keine verbindliche Aussage möglich, da bislang keine Standardmengen HB-Antigen in definierter Zusammensetzung erhältlich waren. Da zudem über den Durchseuchungsgrad der Bevölkerung und über mögliche Einflüsse anderer Serumfaktoren oder veränderter Serumqualitäten nichts bekannt ist, kann ein "Normbereich" auch nicht durch eirie Vielzahl von Bestimmungen an Seren von "Lebergesunden" als Normkollektiv ermittelt werden.

Einfachstenfalls könnte man annehmen, daß ein Serum, das unter Zugrundelegung des „Negativivertes" einen RIA-Faktor von nicht mehr als $1,0 \pm 32 \%$ ergibt, mit einer Sicherheit von mehr als $97,5 \%$ keine mit der Methode nachweisbaren Antigenmengen enthält.

Zur Prüfung dieser Hypothese, eingedenk der unbekannten Empfindlichkeitsschwelle des Verfahrens, haben wir einen "Normbereich" anhand der Ergebnisse von Verlaufsuntersuchungen bei 27 Patienten, die an einer Hepatitis B erkrankt waren, ermittelt. Bei diesen Patienten lag die akute Erkrankung mindestens ein Jahr zurück, die Leberfunktion war bei mehrfachen Kontrollen und unter Anwendung strengster Kriterien normalisiert und histologisch waren keine Zeichen einer Leberentzündung mehr nachweisbar. Unter der Annahme, daß im Verlauf einer Ausheilung der Hepatitis B das Antigen aus dem Serum des Patienten - schwindet, haben wir Mittelwert und 2s-Bereich des "Normbereiches" wie folgt bestimmt: Wenn in der Verlaufsbeobachtung anfänglich hohe RIA-Werte fallende Tendenz zeigten und in mindestens zwei auf- 
Tab. 7

Mittelwert und Standardabweichung des an 27 Patienten, die an Hepatitis B nit transienter Antigenämie erkrankt waren, nach Norinalisierung aller hepatologischen Befunde gefundenen "Norniwertes" für den RIA-Faktor

\begin{tabular}{lllll}
\hline$n$ & 8 & s & $8+2 \mathrm{~s}$ \\
\hline 27 & 1,22 & 0,20 & 1,62 \\
\hline
\end{tabular}

cinanderfolgenden, mehrere Wochen auseinanderliegenden Bestimmungen unter den willkürlich angenommenen Grenzwert 2,0 gefallen waren, wurde der letzte Meßwert als sogenannter ,normalisicrter Wert" in die statistische Analyse cinbezogen. Der niedrigste beobachtete Wert betrug in diesem Kollektiv 0,90, der Höchstwert 1,63 (Tab. 7).

Einfluß der eingesetzten Serummenge und der Menge des zugefügten radioaktiven Antikörpers

Untersuchungen zu dieser Fragestellung wurden an nach der Definition der Methode antigenfreien und antigenhaltigen Seren durchgeführt. Die Erhöhung der eingesetzten Menge Probandenserum bis auf $0,5 \mathrm{ml}$ führte in keinem Falle $z u$ einer statistisch singifikanten Erhöhung der RIA-Faktoren. Auch die Verdoppelung der Menge des radioaktiven Antikörpers (entsprechend $0,2 \mathrm{ml}$ ) ließ die gemessenen RIA-Faktoren nicht signifikant ansteigen. Erst bei größeren Mengen war ein ansteigender Trend erkennbar. Allerdings befanden sich unter den antigenhaltigen Seren keine, deren RIAFaktor im linearen Bereich der Methode lag.

Einflüsse der Inkubationsdauer und Inkubationstemperatur

Dem Bestimmungsverfahren nachteilig ist die lange Inkubationsdauer von $16 \mathrm{~h}$, die eine Bestimmung in eiligen Fällen verhindert. Es wurde daher geprüft, inwieweit Inkubationszeit und Temperaturveränderungen bei der Inkubation die Reproduzierbarkeit der Bestimmungen beeinflussen und ob eine Verkürzung der Inkubationszeit ohne Empfindlichkeitsverlust möglich ist.

\section{Einflus der Inkubationsdauer}

Unter gleichen Bedingungen wurden von 40 antigenhaltigen Probandenseren jeweils 4 Testansätze angefertigt. Zwei der Ansätzc wurden $3 \mathrm{~h}$, dic beiden übrigen $16 \mathrm{~h}$ bei Raumtemperatur inkubiert. Alle Ansätze wurden dann in gleicher Weise weiterbehandelt. Die Ergebnisse wurden einer zweistufigen hierarchischen Varianzanalyse unterzogen, deren Resultate in Tabclle 8 aufgeführt sind.

Während Mittelwerte und Variationskoeffizienten keinen über die „Reproduzierbarkeitsstrcuung“ hinausgehenden Unterschied erkennen lassen, ergab die Bestimmung des F-Wertes mit einem Wert von 3,4 bei $(40,80)$ Freiheitsgraden den Hinweis dafür, daß mehr als zufällige Unterschiede zwischen den beiden Gruppen bestehen. Als mögliche Ursache muß die Verkürzung
Tab. 8

Einfluß der Inkubationsdauer auf die Hölıe der RIA-Faktoren HBAntigenhaltiger Seren, seprüft durch hlerarchische Varianzanalyse (Angaben als RIA-Faktor)

\begin{tabular}{lccc}
\hline & $3 \mathrm{~h}$ & $\begin{array}{c}\text { Inkubatlonsdauer } \\
16 \mathrm{l}\end{array}$ \\
$\begin{array}{l}\text { Anzalil } \\
\text { Doppelbestim- } \\
\text { mungen }\end{array}$ & 40 & & $\begin{array}{c}\text { Insges. } 40 \\
\text { (Vierfachbest.) }\end{array}$ \\
\hline X & 10,3 & 10,8 & 10,6 \\
SSQ & 55,8 & 66,8 & 329,8 \\
S2 & 1,4 & 1,7 & 2,7 \\
VK & $11,4 \%$ & $11,9 \%$ & $15,7 \%$ \\
FG & 40 & 40 & 120 \\
\hline
\end{tabular}

$F=3,4$ bei $(40,80)$ Freilleitsgraden

der Inkubationsdauer angeschen werden. Auch dic Analysc der Einzelwerte deutet auf meht als zufällige Unterschicde: Bei niedrigen RIA-Faktoren im Serum (kleiner als 7,0) wurden nach 3 stündiger Inkubation durchweg niedrigere Wcrtc gefunden als nach 16stündiger Inkubationsdauer. Dagegen ließ ein Vergleich der Meßwerte mit eincm RIA-Faktor über 7,0 keinen gerichteten ' $T$ rend, jedoch eine eindeutig schlechtere Differenzierung in den nach 3 stündiger Inkubation bestimmten Werten erkennen. Nach diesen Ergebnissen ist eine Verkürzung der Inkubationsdauer nicht gerechtfertigt, da mit einer geringeren Nachweisempfindlichkeit gerechnet werden muß.

\section{Einflußs der Temperatur bei verkiirzler Inkubationszeit}

Zur Prüfung der Fragc, inwieweit die durch Verkürzung der Inkubationszcit sinkende Empfindlichkeit des Nachweises durch Temperaturänderungen (Erhöhung) während der Inkubation ausgeglichen werden kann, wurden zwei Untersuchungsgänge vorgenommen: In der ersten Versuchsreihe wurden von 10 antigenhaltigen Seren je 4 Ansätze vorgenommen. Alle Ansätze wurden $3 \mathrm{~h}$ lang inkubiert, zwei davon bei Raumtemperatur, die beiden anderen bei $37^{\circ} \mathrm{C}$. Alle Ansätze wurden anschließend den gleichen Wasch-, Inkubations- und Meßvorgängen unterzogen.

Dic statistische Analyse der errechneten RIA-Faktoren ergab eine deutliche Differenz der Mittelwerte beider Gruppen (10,6 bei Raumtemperatur gegenüber 13,2 bei $37^{\circ} \mathrm{C}$ ) und mit einem $F$-Wert von 10,3 bei $(20,10)$ Freiheitsgraden den eindeutigen Hinweis auf einen Einfluß der Temperaturerhöhung.

In Tabelle 9 sind die Ergebnisse der statistischen Analyse der Gegenprobe aufgeführt. Von 20 Probandenseren wurden je 4 Ansätze vorgenommen. Zwei davon wurden cincr dreistïndigen Inkubation bei $37^{\circ} \mathrm{C}$ unterworfen, die beiden anderen $16 \mathrm{~h}$ bei Raumtemperatur inkubiert. Danach wurden alle Ansätze in gleicher Weisc weiter behandelt.

Die Bcrechnung ergab einen signifjkanten F-Wert von 2,6 bei $(20,40)$ Freihcitsgraden. Dor nicht signifikante Unterschied der Mittelwerte und die differierenden Varianzen weisen darauf hin, daß diese schwache Signifikanz wahrscheinlich auf die bessere Reproduzier- 
Tab. 9

Einfluß der Temperaturerhöhung bei verkürzter Inkubationsdauer auf die Höhe der RIA-Faktoren, bestimmt an HB-antigenhaltigen Patientenseren. Prüfung durch hierarchische Varianzanalyse. (Angabe in RIA-Faktoren)

\begin{tabular}{llcc} 
& \multicolumn{3}{l}{ Inkubationsdauer/Inkubationstemperatur } \\
& $3 \mathrm{~h} / 37^{\circ} \mathrm{C}$ & $16 \mathrm{~h} / 22^{\circ} \mathrm{C}$ & \\
$\begin{array}{l}\text { Anzahl der } \\
\text { Doppelbestim- } \\
\text { mungen }\end{array}$ & & & $\begin{array}{c}\text { Insges. 20 } \\
\text { (Vierfachbest.) }\end{array}$ \\
\hline $\mathrm{x}$ & 20 & 20 & 14,85 \\
SSQ & 14,74 & 14,96 & 226,2 \\
$\mathrm{~s}^{2}$ & 30,7 & 67,0 & 3,8 \\
VK & 1,5 & 3,4 & $13,1 \%$ \\
\hline
\end{tabular}

$F^{\prime}=2,6$ bei $(20,40)$ Freiheitsgraden

barkeit der Werte in der Gruppe nach 3 stündiger Inkubation bei erhöhter Temperatur zurückzuführen ist. Die Analyse der Einzelwerte ergab im Gegensatz zur Untersuchungsgruppe mit verkürzter Inkubationszeit ohne Erhöhung der Inkubationstemperatur keinen Anhalt für einen gerichteten Fehler im Sinne verminderter Empfindlichkeit.

\section{Einfluß von Medikaimenten und Serumfaktoren (Rbeuma- faktor)}

Beeinflussungen der Meßergebnisse des radioimmunologischen HB-Antigennachweises durch Änderungen der Serumqualität, durch bestimmte Serumfaktoren oder durch Medikamente sind bislang nicht berichtet. Auch die hier mit antigenfreien und antigenhaltigen Seren durchgeführten Untersuchungen ergaben keine Hinweise für eine Störanfälligkeit nach in-vitro Zugabe der folgenden Pharmaka $^{1}$ ) in phy'siologischen Mengen: Buscopan, Calcium, Dominal, Effortil, Glucose, Lävulose, Lasix, Makrodex, Neurotrat, Novadral, Tutofusin, Solu-Decortin, Valium.

Gleichzeitiges Vorhandensein des Rheumafaktors im Serum hat keine "falsch-negativen" RIA-Werte zur Folge. Bei an Hepatitis B erkrankten Patienten mit bereits vorher bekanntem positiven Rheumafaktor war das HB-Antigen mit der radioimmunologischen $\mathrm{Me}-$ thode nachweisbar.

Relative Nachweisempfindlichkeit des Verfahrens

Durch simultane Untersuchungen zum Nachweis des HB-Antigens mittels Immunpräzipitationselektrophorese, Komplementbindungsreaktion (KBR) und mit dem radioimmunologischen Testsatz (RIA) wurde im Rahmen von Verlaufsuntersuchungen bei Hepatitis $B$ (42) sowie anhand von Verdünnungsreihen an antigenhaltigen Seren die relative Nachweisempfindlichkeit des Verfahrens überprüft. Bei den Verlaufsuntersuchungen an 35 Patienten war die Antigenämie mit der radio-

1) Buscopan = Butylscopolaminum; Dominal = Prothipendylum; Effortil = 2-Äthylamino-1-(3-hydroxy-phenyl)äthanol hydrochlorid; Lasix $=$ Furosenidum; Makrodex $=$ Dextran $6 \%$ in physiol. $\mathrm{NaCl}$-lösung; Neurotrat $=$ Vitamin $\mathrm{B}_{1}, \mathrm{~B}_{6}, \mathrm{~B}_{12}$, Leberextrakt; Novadral $=$ Norfenefrin- $\mathrm{HCl}$; Solu-Decortin $=$ Prednisolonum succinat. natrium; Valium $=$ Diazepamum; Tutofusin $=$ isotone Elektrolytinfusionslösung.

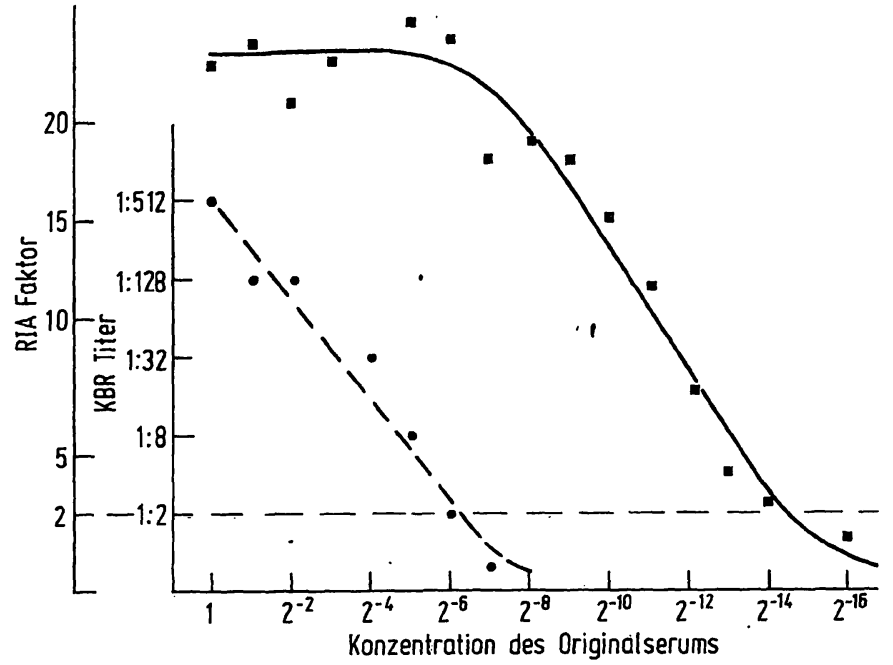

Abb. 1

Empfindlichkeitsvergleich zwischen Komplementbindungsreaktion (KBR, - - und Radioimmunoassay (RIA, - $^{-}$) zum Nachweis von HB-Antigen

immunologischen Methode im Mittel um 30 bis 60 Tage länger nachweisbar (42).

Die relativ höhere Nachweisempfindlichkeit des RIA war auch in 6 Verdünnungsreihen, von denen in $\mathrm{Ab}$ bildung 1 ein Beispiel wiedergegeben ist, erkennbar. Gegenüber der KBR wies das untersuchte radioimmunologische Verfahren in der Regel eine um das 32 bis 64 fache, im Einzelfall bis zu 256 fach höhere relative Nachweisempfindlichkeit auf.

Deutlicher noch als bei den Verlaufsbeobachtungen zeigte sich in den Verdünnungsreihen, daß eine lineare Abhängigkeit zwischen dem Antigengehalt des Serums und dem Wert des RIA-Faktors nur in einem relativ engen Eereich besteht.

\section{Diskussion}

Wie der Ringversuch von SchoвER et al. (43) gezeigt hat, sind Anwendungstechnik und Standardisierbarkeit eines Untersuchungsverfahrens von wesentlicher Bedeutung für die Nachweissicherheit des HB-Antigens im Serum. Durch den Vorzug der digitalen Auswertung erfüllen radioimmunologische Methoden diese erforderlichen Voraussetzungen und sind darüberhinaus der statistischen Fehleranalyse zugänglich. Mit Hilfe der hierarchischen Varianzanalyse ergab die Reproduzierbarkeitsprüfung für den untersuchten solid-phase Radioimmunoassay einen mit $16 \%$ bei Doppelbestimmungen relativ hohen Wert. Es handelt sich dabei jedoch um den Gesamtfehler der Methode bei Bestimmungen von Tag zu Tag, Wechsel der Chargen und Chemikalien und gemessen durch Vielfachbebestimmungen an Patientenseren anstelle eines Standardserums. Wie weiter unten noch erläutert werden wird, beeinflußt dieser vergleichsweise hohe VK die qualitative Differenzierungsqualität des Verfahrens jedoch nur in vertretbarem Ausmaß.

Verlaufskontrollen und Meßergebnisse an /Verdünnungsreihen zeigen jedoch, daß selbst semiquantitative. Aussagen über den Antigengehalt eines Serums nur in 
einem eng begrenzten linearen Bereich möglich sind. Bereits bei einem RIA-Faktor von etwa 10,0 bis 15,0 kann es zu einer Plateaubildung kommen, wobei die Höhe des Plateaus wahrscheinlich weniger von der aktuellen Antigenkonzentration im Serum als von der Antikörperbeschichtung der Polypropylenröhrchen und möglichen Unterschieden in der Relation der Antikörperuntergruppen y und $\mathrm{d}$ abhängig ist.

So problematisch es erscheinen mag, von einem „Normwert" oder "Normbereich" zu sprechen, wird man in der täglichen Diagnostik jedoch nicht um Verwendung eines solchen oder ähnlichen Begriffes vorbei kommen. Bislang wurde nach Literaturangaben derart verfahren, daß ein Serum dann als antigenhaltig bezeichnet wurde, wenn die meßbare Impulsrate des fraglichen Teströhrchens den 3 s oder gar 5s-Bereich des "Negativwertes" der jeweiligen Serie überschritt $(44,45,46)$. Ausgehend von der allgemein gültigen Annahme, daß es nach Ausheilung der überstandenen Virushepatitis zu einem Erlöschen der Virämie und bleibender Immunität kommt, halten wir es für gerechtfertigt, anzunehmen, daß Seren von Patienten, die eine Hepatitis B überstanden haben, die im akuten Stadium der Erkrankung hohe RIA-Faktoren aufwiesen und im Verlauf der Heilung auf niedrigste Werte abfielen, von allen zugänglichen Möglichkeiten die größte Gewähr dafür bieten, antigenfrei zu sein. Es sind daher für die Bestimmung des "Normwertes" auch nur Seren von den Patienten verwendet worden, bei denen die akute Erkrankung ausreichend lange zurück lag, und bei denen weder funktionell, enzymologisch und morphologisch ein pathologischer Leberbefund zu erheben war. Dieser Personenkreis entspricht der Gruppe von Patienten mit vorübergehender Antigenämie bei akuter Hepatitis B entsprechend der Einteilung von BuumBERG (47).

Die Berechtigung der Annahme wird durch die Beobachtung gestützt, daß bei Bestimmung des „Normwertes" bei einem mittleren Wert von 1,2 ein $s=0,2$ resultierte. Diese Standardabweichung entspricht einem VK von $16 \%$ und ist deshalb bemerkenswert, weil sich daraus schließen läßt, daß die persönliche Variabilität innerhalb des „Normbereiches" gering sein muß.

Trotz des relativ hohen VK der Methode ist die qualitative Differenzierungsqualität der Methode auch im Grenzbereich zufriedenstellend. Es läßt sich aus den mitgeteilten statistisch ermittelten Werte ablesen, daß bei einem wahren Wert einer Serumprobe von 2,0 (entsprechend der Definition: Serum ,gering“ antigenhaltig) mit mehr als $85 \%$ Wahrscheinlichkeit ein RIAFaktor größer als 1,6 zu erwarten ist. Liegt der wahre Wert einer Serumprobe bei 2,3, dann beträgt die Wahrscheinlichkeit mehr als $97,5 \%$. Andererseits beträgt die
Wahrscheinlichkeit, daß bei einem wahren Meßwert von 1,2 (entsprechend der Definition antigenfreies Serum) ein höhcrer RIA-Faktor als 1,6 gefunden wird, nur noch $2,5 \%$.

Die Sicherheit der Aussage läßt sich in Grenzfällen durch wiederholte Mehrfachbestimmungen steigern. Diese Forderung ist um so leichter zu erfüllen, als sich bei den jetzt routinemäßig durchgeführten Untersuchungen gezeigt hat, daß nur 3\% aller Meßergebnisse in den als ",kritisch" anzusehenden Bereich fallen. Insgesamt gesehen unterstützen die gefundenen Ergebnisse die Berechtigung des bislang willkürlich gewählten Grenzwertes von ctwa dem Doppelten des „Negativwertes“. Eine endgültige Entscheidung über die Richtigkeit des Vorgehens wird jedoch erst nach Anwendung quantitativer Referenzverfahren möglich sein.

Bei Untersuchung ciniger möglicher Fehlerquellen war nach ersten Ergebnissen anzunehmen, daß die Menge des verwendeten Probandenserums und die Menge des zugefügten radioaktiven Antikörpers Einfluß auf das Meßergebnis haben. Diese Befunde haben sich nicht sicher reproduzieren lassen, so daß angenommen werden kann, da $B$ das Testergebnis weder von der Menge eingesetzten Patientenserums noch von der Menge des radioaktiven Antikörpers beeinflußt wird. Diese Aussage kann als zuverlässig für die wirklich antigenfreien Seren gelten. Bei antigenhaltigen Seren muß jedoch daran gedacht werden, daß eine Exhöhung des RIAFaktors dann denkbar ist, wenn höhere Mengen eines Probandenserums eingesetzt werden, dessen Antigenkonzentration so gering ist, $\mathrm{da} \beta$ die Messung noch im linearen Bereich erfolgt. Für den rein qualitativen Nachweis ist diese Möglichkeit zunächst nicht entscheidend, es wird aber zu prüfen sein, ob nicht auf diese Weise eine verbesserte Empfindlichkeit erzielt werden kann.

Eindeutig und insbesondere im linearen Bereich zeigte sich eine Abhängigkeit der Meßergebnisse von der Inkubationsdauer und der Inkubationstemperatur. Die Analyse der Einzelergebnisse ließ erkennen, daß die Verkürzung der Inkubationsdauer innerhalb des linearen Bereiches einen nach unten gerichteten Trend der Meßergebnisse zur Folge hat. Die an sich wünschenswerte Verkürzung der Inkubationsdauer ist daher nicht ohne weiteres möglich.

Dagegen scheint es nach den vorliegenden Untersuchungen gerechtfertigt, die Dauer der Erstinkubation auf $3 \mathrm{~h}$ zu verkürzen, wenn gleichzeitig die Inkubationstemperatur erhöht wird. Weitere Untersuchungen zur Reaktionskinetik, insbesondere unter Einsatz noch höherer Temperaturen werden daher derzeit durchgeführt (48).

\section{Literatur}

1. Blumberg, B. S., Gerstley, B. J. S., Hungerford, D. A., London, W. T. \& Sutnick, A. J. (1967), Ann. Intern. Med. 66, 924-930. - 2. Krugman, S., Giles, J. P. \& Hammond, J.
(1967), J. Amer. Med. Ass. 200, 365-373. - 3. Окосні, K. \& Musakami, S. (1968), Vox Sang. 15, 374-384. - 4. Prince, A. M. (1968), Lancet II, 462-463. - 5. PrINCE, A. M. (1968), 
Proc. Nat. Acad. Sci. USA 60, 814-821. - 6. AlmerdA, J. D. \& Waterson, A. P. (1971), Internist 11, 73-77. - 7. Berg, P. A. (1972), Klin. Wochenschr. 50, 125-138. - 8. Blumiberg, B. S., Melartin, L., Guinto, A. \& Werner, B. (1966), Amer. J. Hum. Genet. 18, 594-608. - 9. DöLLE, W. (1971). Deut. Med. Wochenschr. 96, 257-258. - 10. Gocke, D. J., Greenberg, H. B. \& KAVEY, N. B. (1969), Lancet II, 248-249. - 11. KABOTH, U., Schober, A., ARndt, H. J., Vido, J., Selmair, H., Gallasch, E., Verna, P., Thomssen, R. \& CReutzfeldt, W., (1970) Deut. Med. Wochenschr. 95, 2157-2165. - 12. KRassnitzKY, O., Pesendorfer, S. \& Wewalka, F. (1970), Deut. Med. Wochenschr. 95, 249-253. - 13. MüLleR, R. \& DeIChER, H. (1971), Verh. Deut. Ges. Inn. Med. 77, 1185-1186. - 14. MülLER, R., KRETH, H. W. \& DeIcher, H. (1971), Deut. Med. Wochenschr. 96, 1268-1274. - 15. MülleR, R., WonNigkeit, K:, BAhLmann, J., Schmidt, F. W., Pichlmayr, R., Ostertag, H. \& Deicher, H. (1971), Klin. Wochenschr. 49, 768-769. - 16. Müller, R., Kalden, J. R., Baruth, B. \& Deicher, H. (1972), Deut. Med. Wochenschr. 97, 369-375. - 17. Prince, A. M., Hargrove, R. C., Szmuness, W., Cherubin, C. E., Fontana, V. J. \& Jeffries, G. H. (1970), N. Engl. J. Med. 282, 987-991. 18. Prince, A. M., Metselaar, M., Kafuko, G. W., Mukwaya, L. G., LING, C. M. \& OVERBY, L. R. (1972), Lancet II, 247-250. 19. Selmair, H., Vido, J., Kaboth, U., Schober, A. (1971), Deut. Med. Wochenschr. 96, 1908-1911. - 20. SCHNEIDER, W. \& FröhlICH, CH. (1972), Diagnostik 5, 213-215. - 21. BARKER, L. F. (1971), Vox Sang. 20, 457-458. - 22. Gocke, D. J. \& Howe, C. (1970), J. Immunol. 104, 1031-1032. 23. KLEX, S. (1972); Deut. Med. Wochenschr. 97, 1547-1549. 24. Kohn, J. \& Morgan, J. D. (1971), J. Clin. Pathol. 24, 673 bis 674. - 25. Pesendorfer, F., Krasnitzky, O. \& Wewalka, F., (1970), Klin. Wochenschr. 48, 58-59. - 26. Prince, A. M. \& Burke, K. (1970), Science 169, 593-595. - 27. Purcell, R. H., Holland, P. V., Walsh, J. H., Wong, D. C., Morrow, A. G. \&
Chammock, R. M. (1969), J. Infect. Dis. 120, 383-386. - 28. World Health Organisation Memoranda (1970), Bull. World Health Organis. 42, 957-992. - 29. ThuLstrup, H. \& DrbKJaER, E. (1970), Vox Sang 19, 234-238. - 30. AACH, R. D., Grisham, J. W. \& Parker, Ch. W. (1971), Proc. Nat. Acad. Sci. USA 68, 1056-1060. - 31. BARKER, L. F. (1971), Vox Sang 20, 457-458. 32. Catt, K. \& Tregear, G. W. (1967), Science 158, 1570-1571. 33. Coller, J. A., Millman, J., Halbherr, Th. C. \& Blumiberg, B. S. (1971), Proc. Soc. Exp. Biol. Med. 138, 249-257. 34. Hollinger, F. B., Vorindan, V. \& Dreesman, G. P. (1971), J. Immunol. 107, 1099-1111. - 35. ZerdLer, U., Dercher, H. \& MÜlleR, R. (1973), Nuc-compact 4, 5. - 36. World Health Organisation Memoranda (1970), Bull. World Health Organis. 42, 957-992. - 37. Overby, L. R., Mrller, J. P., Smith, J. D., Decker, R. H. \& Ling, Ch. M. (1973), Vox Sang 24, $102-113$. 38. Catt, K. \& Tregear, G. W. (1967), Science 158, 1570-1571. 39. BürtneR, H. (1967), diese Z. 5, 41-48. - 40. Mendenthal, W. (1968), Introduction to linear models and the designs and analysis of experiments. Wadsworth Publ. Comp. Belmont Calif. 41. WALtek, E. (1970), Statistische Methoden, Springer-Verlag, Berlin-Heidelberg-New York. - 42. Müller, R., Maess, J., Witrwer, W., Zeidler, U. \& Deicher, H. (1973), Verh. Deut. Ges. Inn. Med. (im Druck). - 43. Schoвer, A., Kaboth, U., Thomsen, R. (1971), Deuț. Med. Wochenschr. 96, 1628-1632. 44. Ginsberg, A. I., Conrad, M. E., Bancroft, W. H., Ling, Ch. M. \& Overby, L. R. (1972), N. Engl. J. Med. 286, 562-566. 45. Prince, A. M., Metselaar, D., Kafuko, G. W.; Mukwaya, L. G., LING, Ch. M. \& Overbx, L. R. (1972), Lancet II, 247-250. 46. Ling, CH. M. \& Overby, L. R. (1972), J. Immunol. 109, 834-841. - 47. Blumberg, B. S. (1972), in: Progress in Liver Disease (Popper, H. \& SchaffNer, S. eds.) 3 ed., p. 16, Grune \& Stratton, New York-London. - 48. ZEIDLER, U., HuLTSCH, E., Dẹicher, H., Kuse, M. \& MülleR, R. (1973), in Vorbereitung.

Prof. Dr. U. Zeidler $\mathrm{MHH}$

3 Hannover-Kleefeld Karl-Wiechert-Allee 9 